\title{
Unrecognized Dural Tear During Transforaminal Percutaneous Endoscopic Lumbar Discectomy
}

\author{
Soo Young Choi, Yong Ahn \\ Department of Neurosurgery, Gil Medical Center, Gachon University College of Medicine, Incheon, Korea
}

Corresponding Author:

Yong Ahn, MD, PhD

Department of Neurosurgery, Gil Medical

Center, Gachon University College of

Medicine, 21, Namdong-daero 774 beon-gil,

Namdonggu, Incheon 21565, Korea

Tel: $+82-32-460-3304$

Fax: +82-32-460-3899

E-mail: ns-ay@hanmail.net

Received: June 21, 2020

Revised: August 14, 2020

Accepted: September 7, 2020
As transforaminal percutaneous endoscopic lumbar discectomy (PELD) becomes more aggressively implemented, the risk for dural tears may increase and lead to severe neurological sequelae. Diagnostic and management strategies for these situations, however, have not been established. This report describes an unrecognized dural tear during transforaminal PELD. A 38-year-old woman sustained an unrecognized dural tear during transforaminal PELD at the L4-5 level. Postoperative radicular symptoms were initially misdiagnosed as recurrent lumbar disc herniation. Despite revision PELD, symptoms were not resolved. The patient underwent endoscopic exploration at the authors' clinic to examine the cause of radicular pain, which revealed a small dural tear and nerve root entrapment at the lateral aspect of the dura. Open repair using interbody fusion was performed at the L4-5 level. The dural tear was primarily closed and the pinched nerve root was relieved. The patient's pain symptoms improved, and she was discharged after adequate wound care. Although PELD is an effective and safe minimally invasive technique, incidental dural tears can occur. Surgeons should be aware of the risks for dural damage and have comprehensive knowledge of clinical features. Early diagnosis with a high level of suspicion is essential to preventing long-term neurological sequelae.

Key Words: Discectomy, Dural tear, Endoscopic, Percutaneous, Transforaminal

\section{INTRODUCTION}

Dural tear during spine surgery is clinically common and one of several critical complications. The prevalence of dural tears in lumbar spine surgery is approximately $1.8 \%$ to $17.4 \%{ }^{2)}$. Sometimes, dural tears may cause clinical sequelae and also lead to medico-legal problems ${ }^{3)}$.

Because minimally invasive spine surgery is a leading trend in the field of spinal treatment, endoscopic spine surgery (ESS) is widely used to treat degenerative lumbar disc disease. Percutaneous endoscopic lumbar discectomy (PELD) is an endoscopic technique that is considered safe and effective, with the advantage of less trauma to healthy tissues. Theoretically, the risk for dural tear is lower in endoscopic discectomy due to minimal retraction of the dural sac. However, as selective endoscopic discectomy for extruded lumbar disc herniation $(\mathrm{LDH})$ is widely performed, dural tears can occur ${ }^{12)}$, and dural damage is becoming a major clinical issue in ESS. The present article describes a case of an unrecognized dural tear during
PELD and discusses the clinical characteristics of this unique complication.

\section{CASE REPORT}

A 38-year-old woman presented with right buttock pain radiating to her right lower extremity. She underwent right transforaminal PELD 2 months previously at another hospital. Preoperative magnetic resonance imaging (MRI) revealed moderate right central disc protrusion at the $L 4-5$ level. According to operative records from the previous hospital, selective endoscopic discectomy after a percutaneous transforaminal foraminoplastic approach was performed. The report stated that the herniated disc fragment was completely removed under endoscopic visualization. However, the patient's complaints of pain radiating down to the right lower extremity persisted after surgery. Despite conservative management, her symptoms did not resolve. She then underwent repeat PELD 1 month later for suspected recurrent LDH. The surgeon removed more disc fragments and confirmed neural decompression on the endo- 
scopic view. A few days later, her back pain and right lower extremity pain worsened, and she visited the authors' spine clinic.

The patient experienced right lower extremity radiculopathy, with a visual analog score (VAS) of 9 in the $L 4$ and $L 5$ dermatome areas. A straight leg raise test could not be performed due to severe radiculopathy. Motor power of right ankle dorsiflexion and the great toe in extension was grade 4.

Plain radiography revealed spondylolisthesis L4 on L5 and disc space narrowing at L4-5. The repeat MRI 2 months after the operation revealed a right-sided central disc protrusion without signs of infection, but the high signal intensity in the intradiscal space and the small defect point at the right subarticular zone were suggestive of dural tears at the L4-L5 level (Fig. 1). However, no definite evidence of dural tear was found on the imaging studies. Therefore, under local anesthesia, the patient underwent transforaminal endoscopic exploration of the previously operated site to confirm the presence of any recurrent LDH or dural defect. Intraoperative findings revealed a small dural defect in the subarticular zone without a definitive recurrent disc fragment. Perfusion pressure applied to the dura

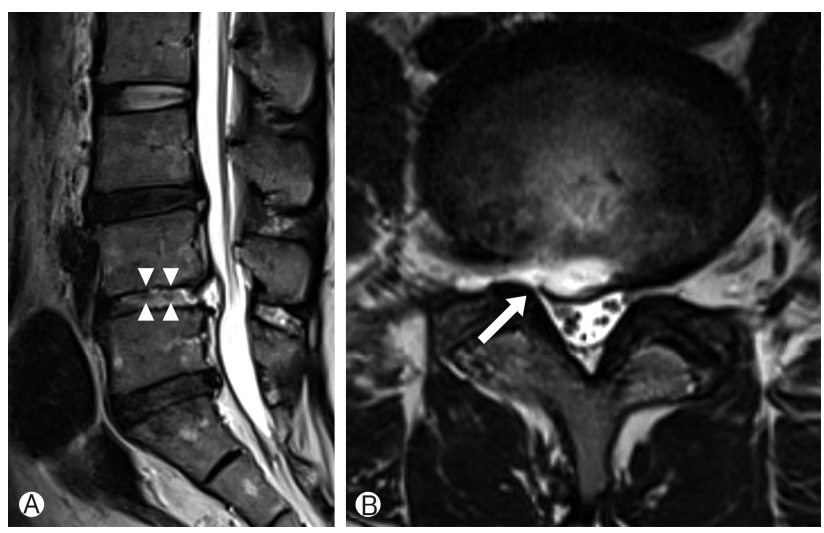

Fig. 1. Magnetic resonance imaging (MRI) findings on admission. (A) T2-weighted sagittal image revealing mild disc protrusion with hyperintense signal changes in the disc space at the level of L4-5 (arrowheads). (B) T2-weighted axial image revealing intradiscal high signal intensity and small defect point at the right subarticular zone suggestive of dural tears (arrow). elicited an unusual muscle twitch and spasm in her leg and severe headache occurred. Primary repair of the defect was not possible. After removing a small amount of disc and scar tissue, the dural tear was sealed using gel foam and fibrin glue. However, the patient's radicular symptoms did not improve despite endoscopic sealing of the dural defect. It was eventually decided to perform open revision surgery for primary repair of the dural defect and stabilize the segment. After a paramedian skin incision, total facetectomy and transforaminal decompression were performed under microscopic visualization. We found a dural tear filled with fibrin glue applied in the endoscopic exploration (Fig. 2(A)). After removing the glue material, a tethered the nerve root and cerebrospinal fluid (CSF) leakage through the defect was found (Fig. 2B). The pinched nerve root was released, and the dural defect was directly repaired using 6-0 prolene and fibrin glue (Fig. 2(C). Finally, further discectomy and interbody fusion were performed, followed by percutaneous pedicle screw fixation at the $L 4-5$ segment. This procedure was performed 2 months after the initial transforaminal PELD. After the final surgery, her right buttock and low extremity pain improved, with VAS score decreasing from 9 to 1 . She was discharged without any neurological sequelae.

\section{DISCUSSION}

A dural tear is a common complication of microscopic open lumbar spinal surgery. In contrast, dural damage after endoscopic lumbar spinal surgery is rare ${ }^{4}$. Most dural tears can occur in the disc removal step, which often occurs when adhesion due to a previous operation or the presence of massive disc herniation $^{2)}$. It is challenging to detect dural injury intraoperatively because the endoscopic surgical field is usually filled with irrigation fluid. The positive pressure of the irrigation fluid may prevent the rootlet from being exposed during PELD"). Therefore, it is difficult to detect small dural tears, which can cause significant neurological symptoms or deficits postoperatively. For this reason, precise knowledge of dural damage in PELD is essential to the success of this ESS technique.

The PELD technique has advanced from indirect disc decompression to selective fragment removal and direct decompression ${ }^{6,10)}$. In the era of indirect decompression, the incidence of dural tears was relatively low. However, in the modern concept
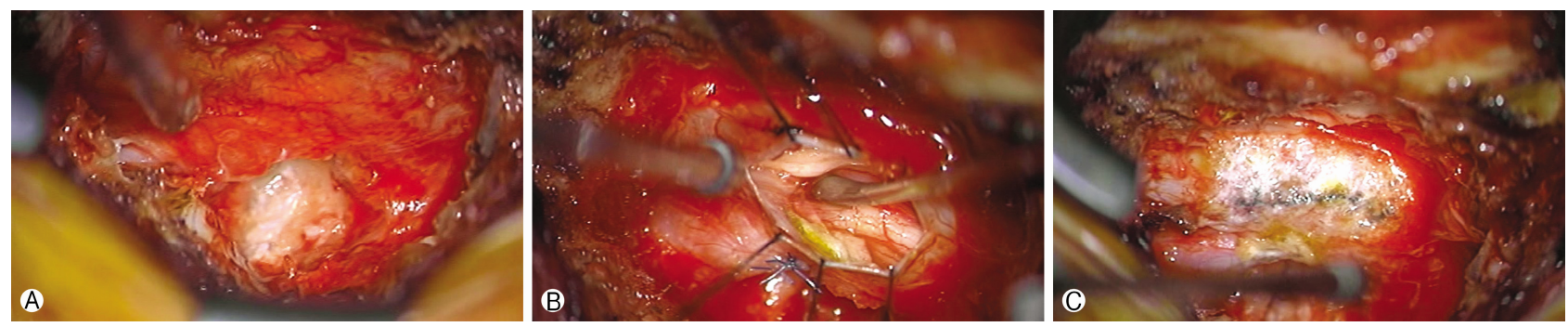

Fig. 2. Operative findings. (A) The initial picture of a dural defect after facetectomy. Note the dural tear filled with fibrin glue applied in the endoscopic exploration. (B) A tethered nerve root and cerebrospinal fluid (CSF) leakage through the defect was found. (C) The dural defect is repaired using 6-0 prolene and fibrin glue. 
of PELD, dural tear have become a unique complication. The risk for dural injury may increase as a surgeon's skill becomes more proficient and pursues a direct and complete dural sac decompression. However, the importance of dural tears in PELD has been overlooked.

Most dural tears occur in either the percutaneous approach or in the selective endoscopic discectomy step. In this case, dural tears may have occurred in the foraminoplastic approach step before the discectomy step. The initial surgeon did not recognize the sign of dural tear from the surgical field or the patient's response. Moreover, the location of the dural tears was the lateral side of the dura, which was not in direct contact with the disc. According to surgical records and the zone of the defect, the dural tears in this case may have occurred during the transforaminal foraminoplastic approach. A definitive diagnosis of dural tear was delayed and, eventually, the patient had to undergo unnecessary revision PELD before the final surgery of open sural repair and interbody fusion surgery.

In water-based ESS, small dural tears may be overlooked due to constant saline irrigation of the surgical field. If unrecognized or untreated, dural tears can develop nerve root impingement or further herniation, causing permanent neurological sequelae or disability. Therefore, a high level of suspicion and knowledge of the clinical features of dural tears is essential.

Intraoperative dural damage can be detected by direct visualization of the dural defect. The nerve root can be exposed or herniated through the dural defect, such as neural herniation, in open surger ${ }^{4,8,9)}$. In that case, the patients frequently complain of back pain, leg numbness, headache, or voiding sensation. Sometimes, muscle twitching can be shown as the irrigation saline elevates intrathecal pressure. Typical CSF leakage or wound swelling is rarely detected because the surgical field is always filled with irrigation saline ${ }^{7)}$.

If the patient complained of severe recurrent or persistent radicular pain after PELD, dural tears should be suspected. In particular, when the nature of the pain is atypical or resembles non-dermatomal electric shock, it may be a sign of nerve root impingement due to the dural defect. L-spine MRI should be performed, and the clinical course should be carefully examined.

There may be some typical radiographic findings of dural tears in PELD. CSF collection in the disc space may manifest as a high signal intensity on T2-weighted MRI ${ }^{7}$. Any entrapment of the nerve root by the dural membrane may be observed on sagittal MRI. Sometimes, a defect in the dural layer may be detected. However, in many cases, the dural defect cannot be identified in imaging studies. Therefore, radiographic findings should be interpreted with a careful review of the clinical characteristics and course. In our case, we found a small dural defect at the lateral aspect of the dura. However, the previous surgeon did not recognize the dural defect on postoperative MRI. In cases of unrecognized or untreated dural tear, chronic neuropathic pain, cauda equina syndrome, or long-term neurological sequelae may develop.

The risk for dural tears may increase in cases of recurrent, migrated, or high-canal compromising LDH. Sophisticated dis- section of the tight adhesion between the disc material and dural membrane may be difficult in these cases. Regarding the surgeon factor, aggressive dissection and decompression may increase the risk for dural tears. In their learning curve or during the training period, surgeons may experience difficulty with appropriate instrumental manipulation in the endoscopic surgical field.

In most cases, dural tears in PELD can be avoided by using preventive strategies. First, accurate knowledge of the endoscopic surgical anatomy is essential. The anatomical layer, including the dural sac, epidural fat, posterior longitudinal ligament, torn annulus, and herniated disc material, should always be distinguished. The barrier or space between the dural sac and herniated disc should be precisely dissected before selective discectomy. Second, the surgeon should be highly familiar with the features of the endoscopic instruments. Delicate instrumental manipulation should be essential in two-dimensional endoscopic vision. Finally, the endpoint of the procedure should be free mobilization and pulsation of the neural tissue. The direct and full exposure of neural tissues may be challenging and dangerous in a risky case. Instead, restoration of dural pulsation and mobilization may be useful, even though some adhered disc materials remain.

Despite the best efforts of the surgeon, an incidental dural tear may occur. Early detection and adequate management are essential to prevent long-term sequelae ${ }^{6,11)}$. In most cases, primary and water-tight closure is impossible in a narrow endoscopic surgical field. If the defect is small and the nerve root is within the dural sac, a precise surgical tamponade using shielding materials and fibrin glues can be useful. The patient's response should be carefully monitored to determine whether any signs of dural tear occurs. If the nerve root is herniated during the procedure or the patient exhibits signs of dural tear, open primary repair can be considered. The patient should be maintained on bed rest and close monitoring for $72 \mathrm{~h}$ postoperatively.

\section{CONCLUSION}

Dural tear is a unique complication of PELD. As this minimally invasive technique becomes increasingly accessible and practical, the incidence of unexpected dural damage is also likely to increase. Unrecognized dural tear(s) can cause significant disability and/or neurological deficits. Therefore, surgeons should carefully consider the clinical characteristics and management "know-how" of accidental/incidental dural injury during PELD.

\section{ACKNOWLEDGMENTS}

The authors would like to thank Kyung-Hee Jang and Uhn Lee for their support and assistance.

\section{REFERENCES}

1. Ahn Y, Lee HY, Lee SH, Lee JH: Dural tears in percutaneous endoscopic lumbar discectomy. Eur Spine J 20(1):58-64, 2011 
2. Bosacco SJ, Gardner MJ, Guille JT: Evaluation and treatment of dural tears in lumbar spine surgery: A review. Clin Orthop Relat Res 389:238-247, 2001

3. Goodkin R, Laska LL: Unintended "incidental” durotomy during surgery of the lumbar spine: Medicolegal implications. Surg Neurol 43:4-12, 1995

4. Hasegawa K, Yamamoto N: Nerve root herniation secondary to lumbar puncture in the patient with lumbar canal stenosis: A case report. Spine (Phila Pa 1976) 24(9):915-917, 1999

5. Jones AA, Stambough JL, Balderston RA, Rothman RH, Booth RE Jr: Long-term results of lumbar spine surgery complicated by unintended incidental durotomy. Spine (Phila Pa 1976) 14(4): 443-446, 1989

6. Kambin P, O'Brien E, Zhou L, Schaffer JL: Arthroscopic microdiscectomy and selective fragmentectomy. Clin Orthop Relat Res 347:150-167, 1998

7. Khan MH, Rihn J, Steele G, Davis R, Donaldson WF 3rd, Kang JD, et al: Postoperative management protocol for incidental dural tears during degenerative lumbar spine surgery: A review of 3,183 consecutive degenerative lumbar cases. Spine (Phila Pa 1976)
31(22):2609-2613, 2006

8. Nishi S, Hashimoto N, Takagi Y, Tsukahara T: Herniation and entrapment of a nerve root secondary to an unrepaired small dural laceration at lumbar hemilaminectomies. Spine (Phila Pa 1976) 20(23):2576-2579, 1995

9. Töppich HG, Feldmann H, Sandvoss G, Meyer F: Intervertebral space nerve root entrapment after lumbar disc surgery: Two cases. Spine (Phila Pa 1976) 19(2):249-250, 1994

10. Tsou PM, Yeung AT: Transforaminal endoscopic decompression for radiculopathy secondary to intracanal noncontained lum bar disc herniations: outcome and technique. Spine J 2(1):41-48, 2002

11. Wang JC, Bohlman HH, Riew KD: Dural tears secondary to operations on the lumbar spine. Management and results after a two-year-minimum follow-up of eighty-eight patients. J Bone Joint Surg Am 80(12):1728-1732, 1998

12. Yeung AT, Tsou PM: Posterolateral endoscopic excision for lumbar disc herniation: Surgical technique, outcome, and complications in 307 consecutive cases. Spine (Phila Pa 1976) 27:722-731, 2002 